\title{
VARYING PATTERNS OF THE LOBAR BRANCHES OF THE PULMONARY ARTERY \\ A STUDY OF 524 LUNGS AND LOBES SEEN AT OPERATION ON 426 PATIENTS \\ BY
}

\author{
RICHARD A. S. CORY AND EDWARD J. VALENTINE \\ From King George V Jubilee Memorial Sanatorium, Liguanea, Jamaica, W.I.
}

(RECEIVED FOR PUblication JUNE 23, 1959)

This study arose from our wish to determine with reasonable accuracy what variations in the pattern of the pulmonary artery and its lobar branches might be found in a series of pulmonary resections carried out on a predominantly coloured population group.

To this end careful diagrams have been made after operation on all patients treated by lung resection in the King George V Jubilee Memorial Sanatorium in Jamaica between April, 1955, and May, 1959. The operator (one of us) used to record in the form of a sketch the arterial anatomy found at each dissection.

In the period under review 426 patients have been submitted to some form of resection, and it has been possible to demonstrate with accuracy the arterial pattern found in a total of 524 lungs or lobes. Table I shows the actual types of resection performed on the 426 patients.

TABLE 1

TYPES OF RESECTION

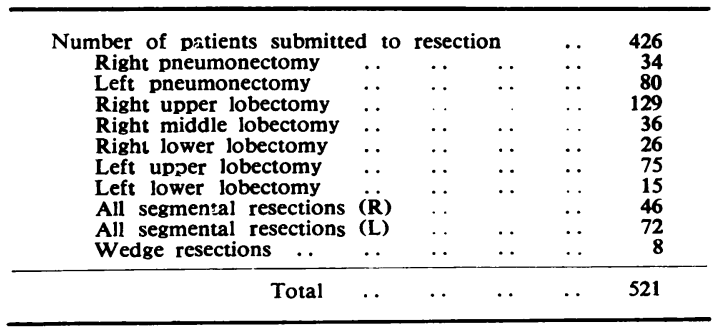

Of the total of 521 resections performed 513 were anatomical (lung, lobe, or segment), and eight were not anatomical (wedge).

In the pure wedge resections demonstrations of hilar arterial anatomy have not been attempted, but in the 418 patients submitted to some form of anatomical resection the primary or secondary surgical hilum has been accurately demonstrated in 524 lungs or lobes.
Table II shows the actual demonstrations that have been found possible, and it is upon these that this paper has been based.

TABLE II

ACTUAL HILA DEMONSTRATED

\begin{tabular}{|c|c|c|c|c|}
\hline $\begin{array}{l}\text { Right primary } \\
\text { Left primary } \ldots \text {. } \\
\text { Right upper lobe (secondary) } \\
\text { Right middle lobe (secondary) } \\
\text { Right lower lobe (secondary) } \\
\text { Left upper lobe (secondary) } \\
\text { Left lower lobe (secondary) }\end{array}$ & $\begin{array}{l}\cdots \\
\cdots \\
\cdots \\
\cdots \\
\cdots\end{array}$ & $\begin{array}{l}\cdots \\
\cdots \\
\cdots \\
\cdots \\
\cdots \\
\cdots\end{array}$ & $\begin{array}{l}\cdots \\
\cdots \\
\cdots \\
\cdots \\
\cdots \\
\cdots\end{array}$ & $\begin{array}{r}34 \\
80 \\
152 \\
51 \\
43 \\
107 \\
57\end{array}$ \\
\hline Total & . & $\cdots$ & $\ldots$ & 524 \\
\hline
\end{tabular}

The number of demonstrations considerably exceeds the number of patients operated upon because a large proportion of the patients underwent more than one resection at the same operation, and in a few a second operation for further resection was required.

Resected specimens were not injected, and on occasions some confusion with regard to the exact branches to individual segments has arisen where resected lobes have been grossly shrunk or distorted by disease. These difficulties have of course been more evident in the case of the upper lobes where the arterial supply is subject to more variation than it is in the middle and lower lobes. Where possible, however, the segmental vessels whose distribution has been accurately ascertained have been noted on the drawings.

We operate in either the lateral or face-down position, but most frequently from the lateral approach. All the drawings therefore have been represented from the surgeon's viewpoint when he is standing posterior to a patient placed in the lateral position, and they illustrate the various hila as seen from the lateral aspect.

\section{The Various Surgical Hila}

Right Primary Hilum (Fig. 1).-The main pulmonary arterial trunk as encountered at right pneumonectomy showed no variations in our 34 
cases. It came out always in the same position, in the angle between the azygos vein above and the superior vena cava in front, and it lay anterior to the bronchus.

Here it gives its main branch to the upper lobe. It may be ligated above this branch, but we have found it simpler to ligate the branch first and the descending stem separately, just below the branch described. This manceuvre keeps the surgeon away from pericardial sleeves which may make more proximal dissection of the artery in benign cases unnecessarily difficult.

Left Primary Hilum (Fig. 1).-As in the case of the right primary hilum, we found no variation in the position of the left main pulmonary artery in the normal two-lobed left lungs in 80 cases. In one case of dextrocardia with a three-lobed left bronchial pattern there was an eparterial bronchus to the upper lobe which lay immediately lateral to the artery, and crossed it from behind forwards, as is the case on the right side (see Fig. 10B). In this dissection the bronchus completely obscured the main pulmonary arterial trunk proximal to its first branch.
In the usual left primary hilum in two-lobed lungs the artery emerges below the aortic arch, and lies wholly above the bronchus until it gives its first branch. Thereafter it runs downwards behind and lateral to the bronchus.

The left main pulmonary trunk is much more के easily ligated above its first branch than is its $\overrightarrow{0}$ counterpart on the right, as it is appreciably $\overrightarrow{-}$ longer and is not apt to be invested with peri- $\vec{\omega}$ cardium at the usual site of ligation.

Right UPPER Lobe (SECONDARY Hilum) (Figs. $\stackrel{x}{\vec{x}}$ 2 and 3).-We were surprised to find no fewer $t$ than 14 variations in the arterial pattern in $152 \stackrel{+}{\mathrm{N}}$ right upper lobes accurately demonstrated. This $\mathcal{G}$ lobe may be supplied by one, two, three, or four 0 segmental vessels. In three cases we found that the artery to the posterior segment arose from the $\vec{\nabla}$ vessel supplying the apical segment of the lower $\mathbb{D}$ lobe (Fig. 3, No. 7, and Fig. 5, No. 5), and in two $\frac{\Phi}{3}$ cases a separate anterior segmental artery arose from the middle lobe supply (Fig. 3, No. 6).

Of the 14 variations, however, only four (Fig. 2, $\overrightarrow{0}$ Types 1-4) can be regarded as at all common. S In the 152 lobes we were able to demonstrate, $\square$

34 right pneumonectomies.

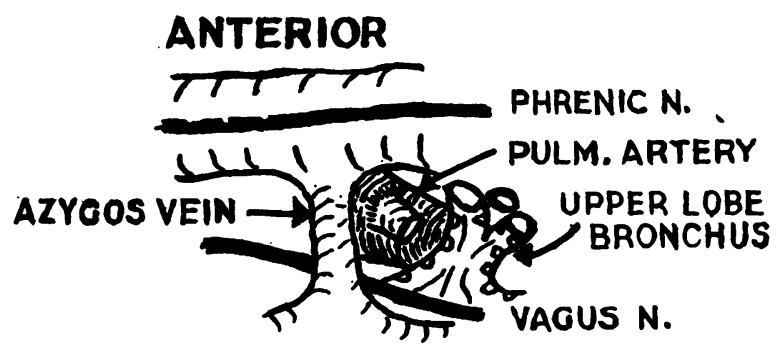

POSTERIOR

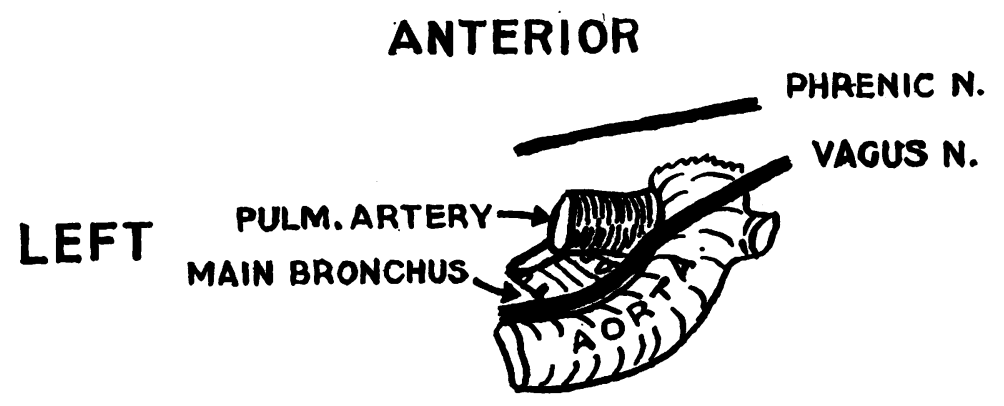

80 left pneumonectomies.

R.C.

Fig. 1.-The primary hilum of the lung. The hila are shown from the lateral aspect with the surgeon standing at patient's back. Anatomy of the pulmonary artery in the primary hilum did not vary. 
these four variations accounted for $91 \%$ of the total.

Type 1 (Fig. 2), which shows two vessels, a single high branch supplying the apical and anterior segments and a low single branch to the posterior segment, was by far the commonest and was found in 91 of 152 lobes $(59.8 \%)$. In Type 2 all the three segmental vessels arise from a single trunk located high and laterally or anterolaterally on the main stem. Twenty-six of the 152 lobes were of this type $(17.1 \%)$.

In Type 3 the posterior segment receives two separate branches. This arrangement was found 13 times $(8.5 \%)$.

Type 4 , which was found nine times $(5.9 \%)$, is a mixture of Types 1 and 2 in that there is a large trifurcated upper branch, and in addition a single branch to the posterior segment. It was our impression that the posterior segment received blood from the upper branch as well as from its own normally located vessel. We were, however, only able to prove this in one-third of the lobes seen with this Type 4 arrangement, and it is possible that in the others the anterior segment may have received two branches, the remaining one going only to the apical segment. This arrangement was not proved in any of the cases.

The remaining types (5-14) of upper lobe arterial patterns are rare, as together they all account for only about $9 \%$ of the lobes demonstrated.

The drawings of these more unusual types in Fig. 3 adequately illustrate the variations that may be found, and emphasize the fact that the surgeon must always be on the look-out for one more artery when he feels that he has already found them all. This extra one is most likely to be an additional one to the posterior segment, or a separate one to the anterior segment. He should also not forget the occasional case with an anterior segmental supply from the middle lobe artery (Fig. 3, No. 6), or the one in which the posterior segment is supplied from the vessel to the apical segment of the lower lobe (Fig. 3, No. 7).

Right Middle Lobe (Secondary Hilum) (Fig. 4).-Fifty-one middle lobes were accurately demonstrated, and in these five variations in the arterial pattern were found. In 25 of the 51 lobes $(49 \%)$ there were two separate arteries, and in 23 , or about another $45 \%$, there was only one. These types, 1 and 2, are the common ones, and account for well over $90 \%$ of all the middle lobe arterial patterns found.

Types 3,4 , and 5 have only one representative each, but they serve to remind us that on rare

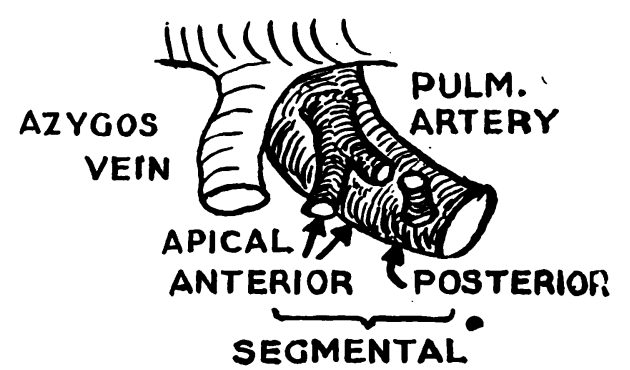

Type 1 , the common type, found in 91 of 152 right upper lobes demonstrated.

Type 2, found in 26 of 152 lobes.
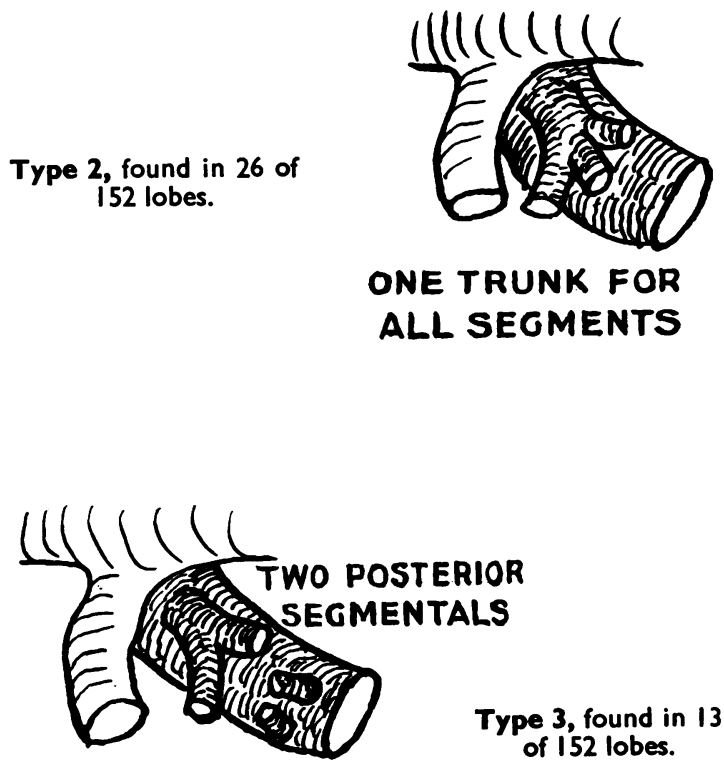

Type 4, found in 9 of 152 lobes.

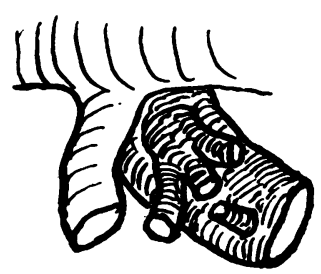

R.C.

FIG. 2.-Right upper lobe ( 152 lobes demonstrated). The above four types accounted for $91 \%$ of all the right upper lobe pulmonary arterial patterns found. All seen from behind. 
Type 5

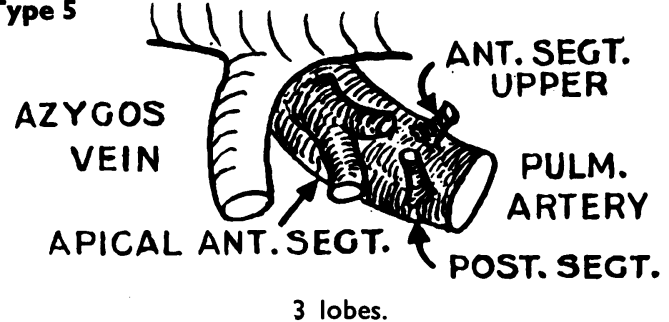

Type 6

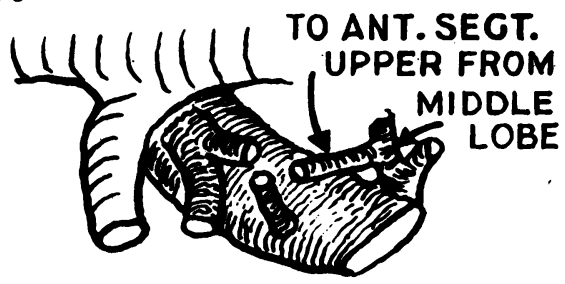

2 lobes.

Type 7

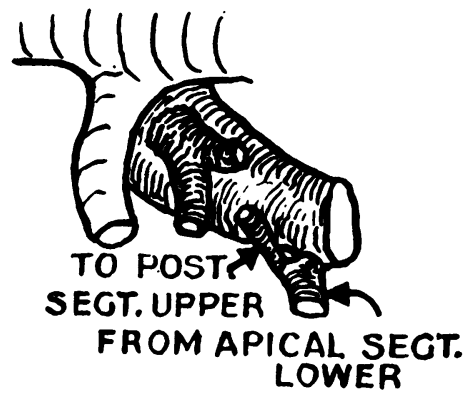

I lobe.

Type 8

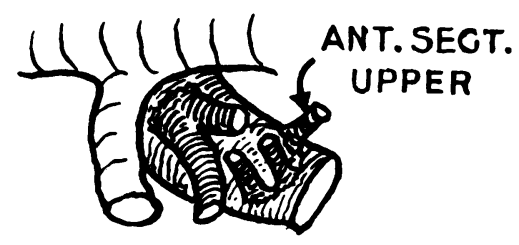

I lobe.

Type 9

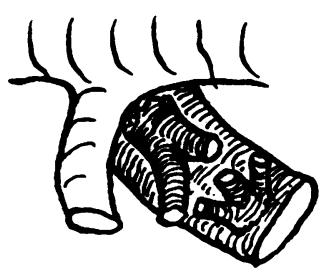

I lobe.

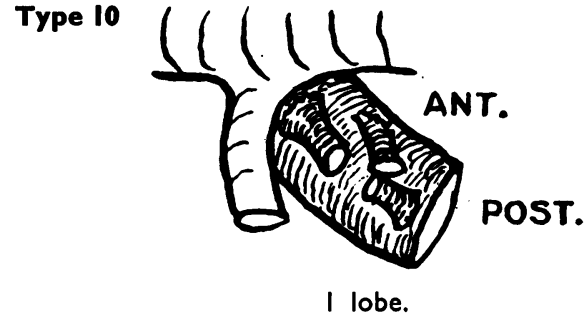

Type

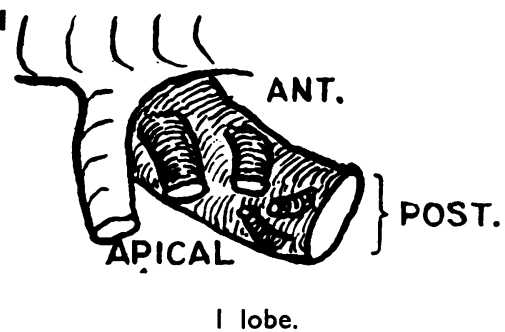

Type 12

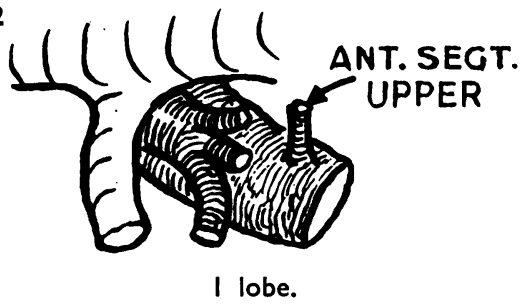

Type 13

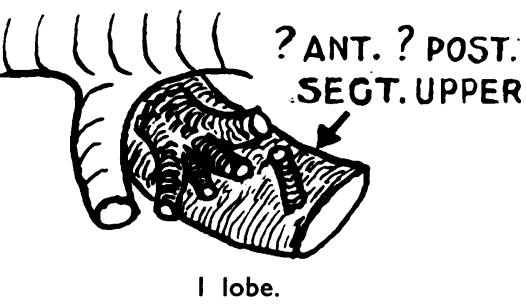

Type 14

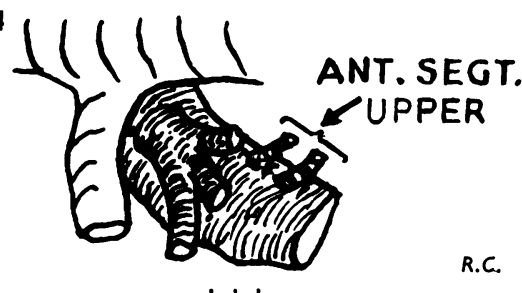

Fio. 3.-Right upper lobe (152 lobes demonstrated) showing the less common pulmonary arterial variations. The above 10 types together $\overparen{\mathscr{Q}}$ account for only $9 \%$ of the right upper lobe pulmonary arterial patterns found. All seen from behind. 
Type I

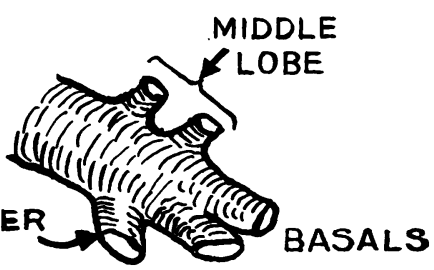

25 of 51 lobes demonstrated.

Type 2

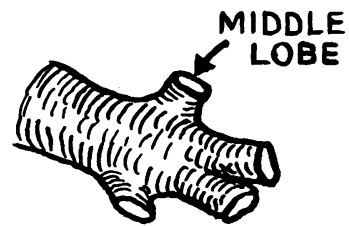

23 of 51 lobes demonstrated.

Type 3

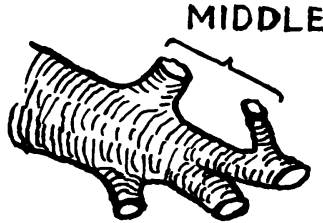

I lobe.
Type 4

MIDDLE

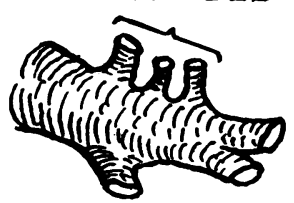

I lobe.
Type 5

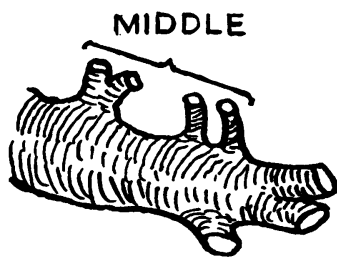

I lobe.
Fig. 4.-Right middle lobe (51 lobes demonstrated). Types 1 and 2 are the common types and together they account for $94 \%$ of all middle lobe pulmonary arterial patterns found. Only $6 \%$ of middle lobes belonged to the three unusual patterns above. All seen from behind.

occasions the middle lobe may receive three separate branches from the descending trunk of the pulmonary artery, or even an ascending vessel from one of the basal segmentals.

We did not find any instance of the middle lobe receiving a vessel from one of the branches to the upper lobe.

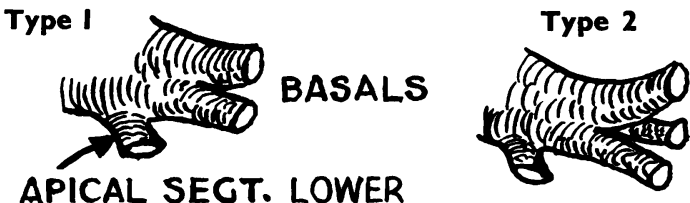

31 of 43 lobes demonstrated $=72 \%$. 4 lobes $=9 \%$.

Type 3

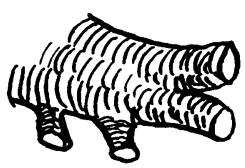

3 lobes.

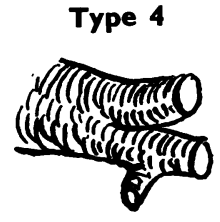

2 lobes.
Type 5

TO POST.SEGT.

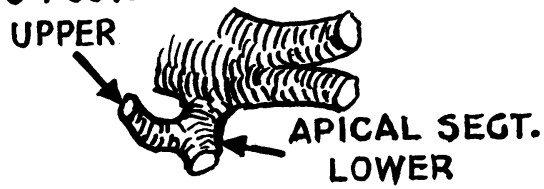

2 lobes

(One other example of this pattern was found at a right upper lobectomy. See Fig. 3.)

\section{Type 6}

POST. SEGT.

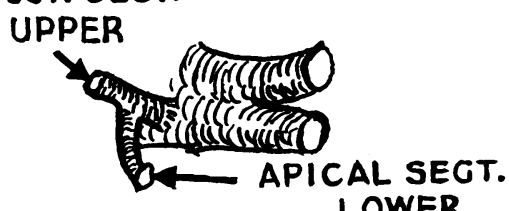

LOWER

I lobe.

R.C.

Fig. 5.-Right lower lobe (43 lobes demonstrated). Types 1 and 2 together account for $81 \%$ of all right lower lobe pulmonary arterial patterns found. All seen from behind.

Right Lower Lobe (Secondary Hilum) (Fig. 5).-Forty-three right lower lobes were demonstrated, and among them six arterial patterns were found. Type 1 comprised 31 of the 43 lobes $(72 \%)$. This is the common type, and shows one artery to the apical segment taking its origin posteriorly from the pulmonary stem just before this breaks into two main terminal divisions, each 
Type I

Type 2

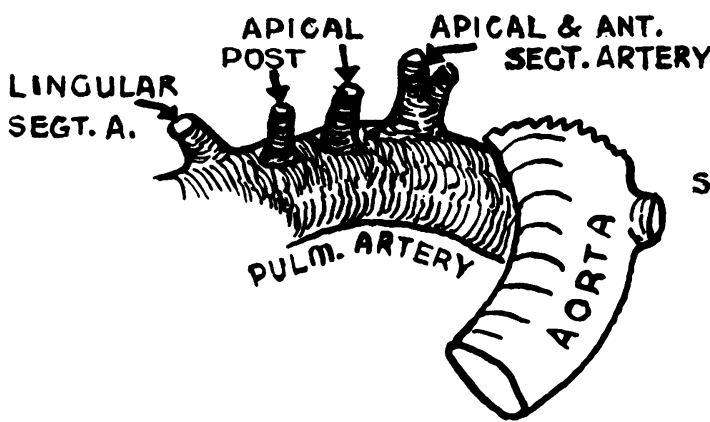

\section{SUB CLAV. ARTERY}

Type 3

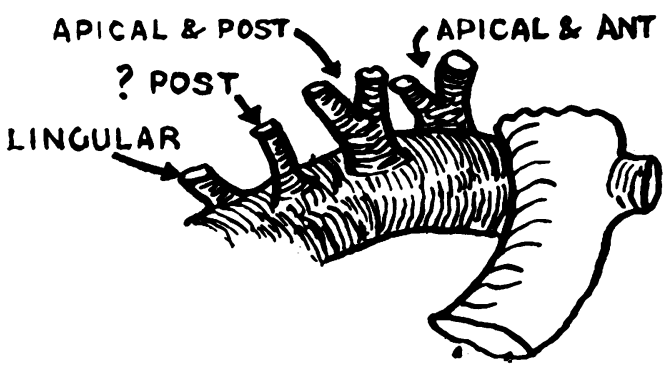

13 of 107 lobes (modified Type 1, in which the apicalanterior artery is more medially placed) $=12 \%$.
Type 4

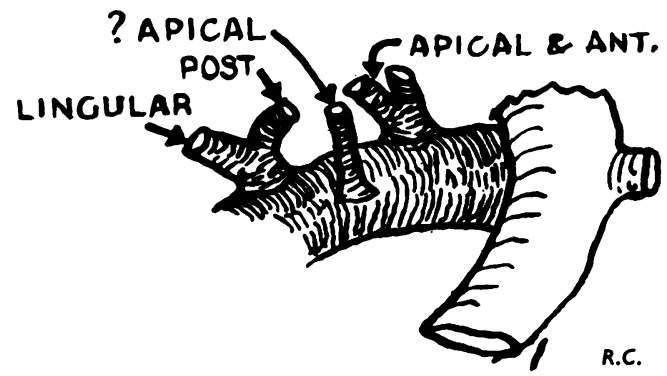

II of 107 lobes (modified Type 2, in which lingular artery gives a large branch to superior segments) $=10 \%$.

Fig. 6 (see also facing page).-Left upper lobe (107 lobes demonstrated).

of which subsequently divides into the vessels to the basal segments.

In Type 2 (four lobes or $9 \%$ ), the artery to the apical segment has the same site of origin, but the terminal trunk divides at once into three instead of two divisions as in Type 1 .

Type 3 (three lobes) shows two separate branches to the apical segment, and two terminals, whereas in Type 4 the only vessel to the apical segment actually arises from the posterior of the two terminal divisions, and not from the descending trunk of the main artery before its bifurcation.

In Type 5 the apical segmental artery gives off the posterior segmental artery to the upper lobe. This arrangement was found twice in lower lobe dissections (43) and once in upper lobe dissections (152).

In the case of the upper lobe dissections it was known to be the sole source of supply to the posterior segment, but in the lower lobe dissections the upper lobe was not sufficiently displayed for us to be sure whether this was also true in these two cases.
Type 6 (Fig. 5) is quite unusual, and comprised only one case. A large artery arose low down laterally on the descending pulmonary trunk, ran directly upwards to the posterior segment of the $\underset{x}{\vec{D}}$ upper lobe, and in its course gave off a branch $\frac{-}{-}$ which was the only vessel supplying the apical 3 . segment of the lower lobe.

LEFT UPPER Lobe (SECONDARY HiLUM) (Figs. 6, 7 , and 8).-One hundred and seven left upper 음 lobes were demonstrated, and 29 arterial patterns $>$ were found.

Although some of the patterns are modifications of others, they nevertheless present a challenge. to the surgeon as being out of the ordinary. $N$ Although recognizing that this lobe is notoriously subject to arterial variations, we were franklyo surprised to find so many in only 107 patients.o There seems to be virtually no truly normal pattern for the left upper lobe, and the branches? to it from the pulmonary artery vary in number 0 from two (seven lobes) to seven (one lobe).

Fig. 6 illustrates the seven types which in our experience have together accounted for slightly over $70 \%$ of the patterns found in our cases. 
Type 5

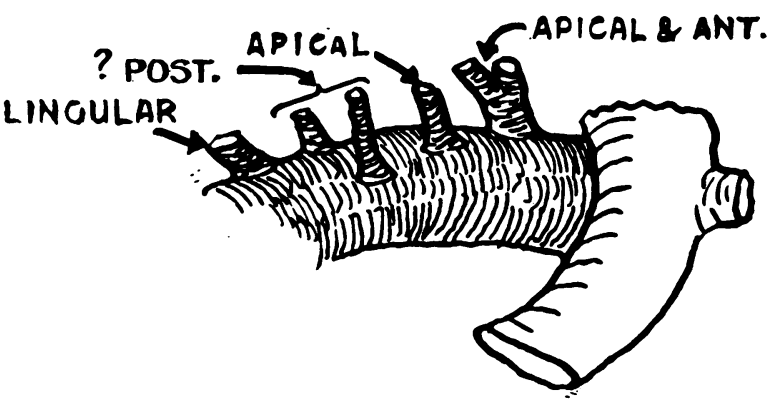

4 of 107 lobes $=3.7 \%$.
Type 6

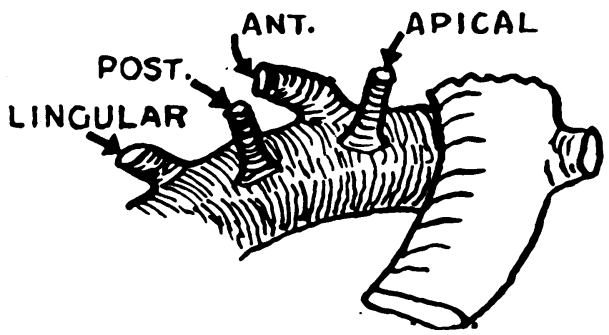

4 of 107 lobes $=3.7 \%$.

Type 7

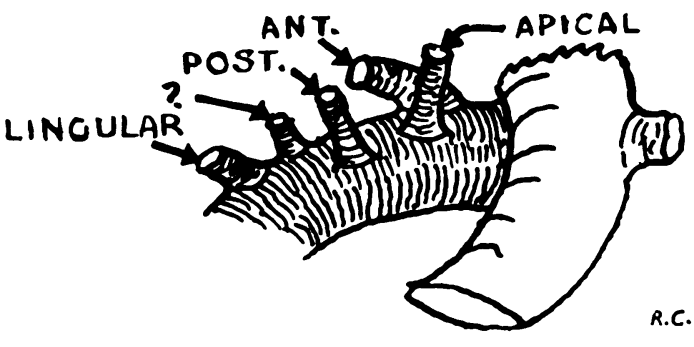

4 of 107 lobes $=3.7 \%$. types account for about $74 \%$ of all the left upper lobe pulmonary arterial patterns found. All seen from behind.

If there is any "normal arrangement" it probably consists of a combination of Types 1 and 3 which together accounted for 49 lobes or about $36 \%$. The essential arrangement shows four arteries, of which the upper three supply the superior segments and the lowest the lingula. The main point of difference between these two types lies in the site of origin of the highest branch.

In Type 1 this is on the anterior or anterolateral surface of the main pulmonary artery, and in Type 2 it is located much more medially and usually at a rather higher level. We have called this an apical and anterior artery, as we have found that it commonly-though probably not invariablysupplies the apical segment, while sending its major branch to the anterior segment. It is in our opinion the counterpart of the highest branch on the right side.

Type 2 is the second commonest, accounting for $14 \%$ in our series. There are three branches, of which the upper always supplies the apical and anterior segments, the second the posterior segment, and the third or lowest the lingula. This distribution is very similar to the common form of supply to the right upper lobe.

Type 4 is a modification of Type 2 , but here the second vessel seems to go to the apical segment, which is also supplied from the high apical- anterior branch, while the posterior segment appears to be supplied by a branch from the lingular artery.

Types $1,2,3$, and 4 together account for about $60 \%$ of the patterns found in our left upper lobes.

There is little doubt about the lingular artery or arteries. Considerable variation is found here also, but it is nearly always possible to be reasonably certain at an operation which vessel supplies the lingula. In between the highest and the lingular branches, however, in lobes with five or more vessels certainty is much more difficult, and on occasions quite impossible.

In Type 5 (Fig. 6), for instance, there is an apical and anterior artery and a well-defined single vessel to the lingula. Between these two lie three others. The upper of these three probably supplies the apical segment, and the other two the posterior segment. At operation, however, where segments are often not identifiable on account of shrinkage and distortion following disease it may be virtually impossible to be certain of the course of any particular vessel. While this uncertainty is of no great moment where lobectomy has to be done, it is of considerable importance in segmental resections, and leaves one wondering how often a remaining segment may have been robbed of a large portion of its pulmonary arterial supply during early dissection 
Type 8

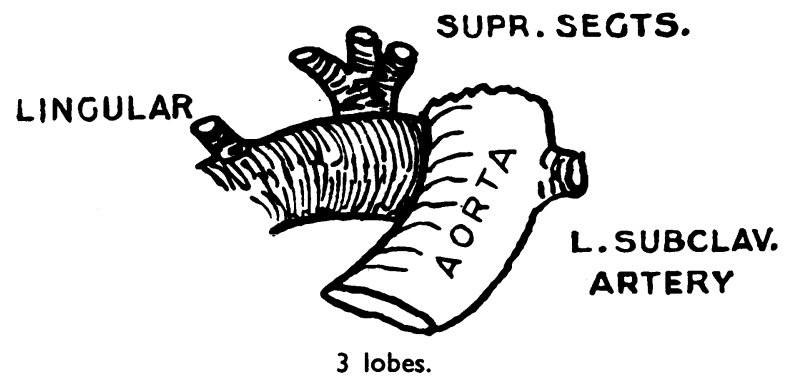

Type 10

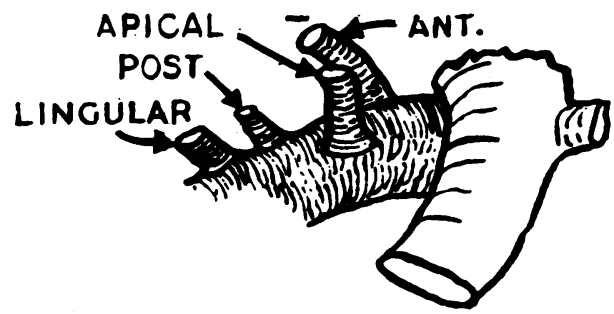

2 lobes (modinied Type 6).

Type 12

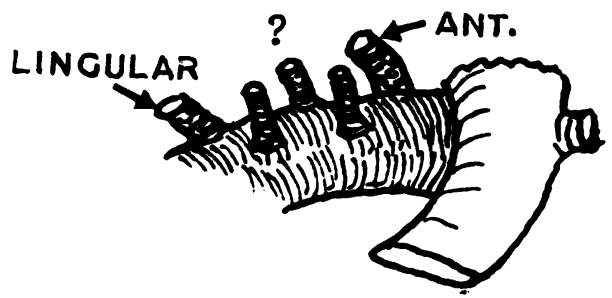

2 lobes (modified Type 5).
Type 9

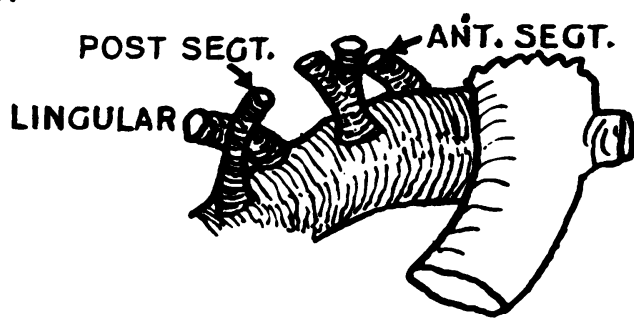

2 lobes.

Type II

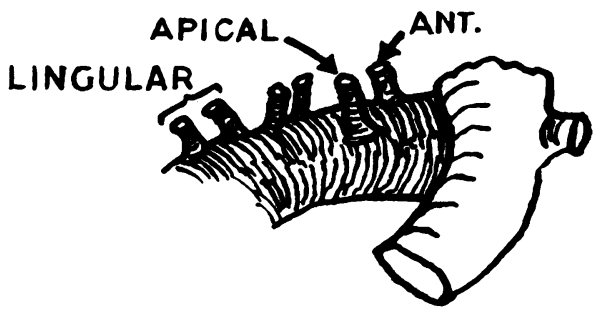

2 lobes. and ligation of the vessels. This thought has caused us considerable concern with segmental resections on the left upper lobe, and, with the many variations we have demonstrated, our concern is growing.

Type 5 shows five vessels. The upper and lower are reasonably certain, but difficulty arises in determining the course of the three in between. Type 6, on the other hand, seems clear-cut. The highest branch arises laterally and supplies the apical segment. The next branch arises anteriorly and goes to the anterior segment. Branch 3 goes to the posterior segment (or posterior portion of
Type 13

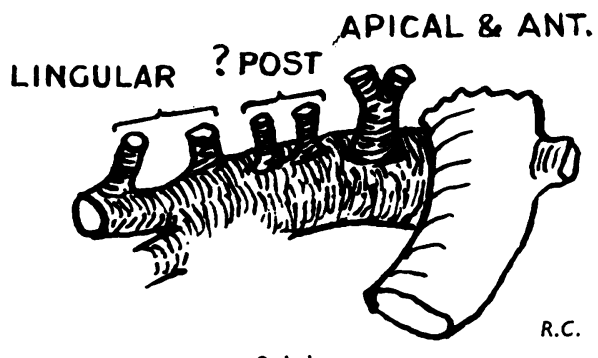

2 lobes.

the apical-posterior compound segment) and the fourth branch is a normal lingular artery.

In Type 7, with five branches, difficulty arises $N$ with branch number 4 . Does it go to the anterior or to the posterior segment? Without injection studies on hundreds of normal lungs that have not been subjected to segmental distortion by disease we do not see clearly how this difficulty is $\stackrel{\mathscr{D}}{+}$ to be accurately resolved. Our dissections have $\square$ revealed 29 differing arterial patterns for the left $\bar{\circ}$ upper lobe, and we frankly admit that we have not $\mathbb{D}$ been able to determine with accuracy the zones of $\mathbb{D}$ supply of many of the vessels found. 
Type 14

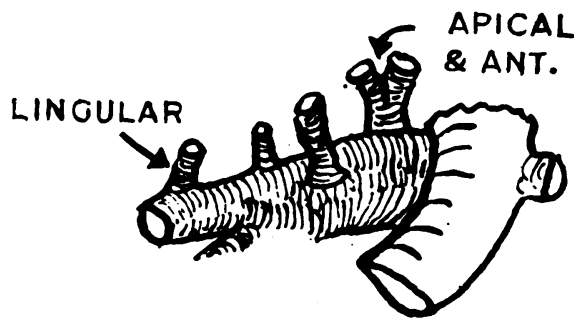

I lobe.

Type 16

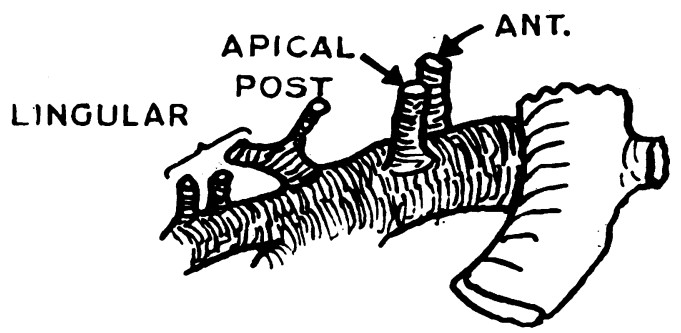

I lobe.

Type 18

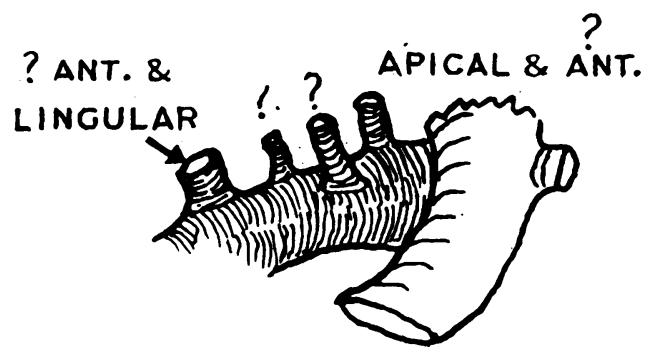

I lobe.
Type 15

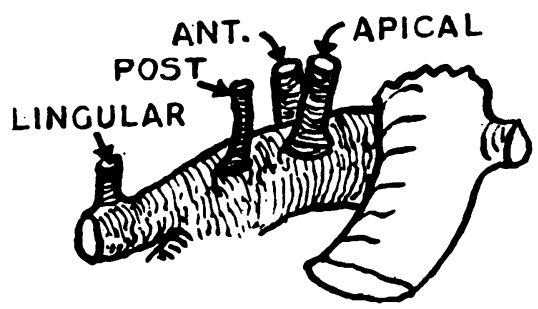

I lobe (modified Type 14).

Type 17

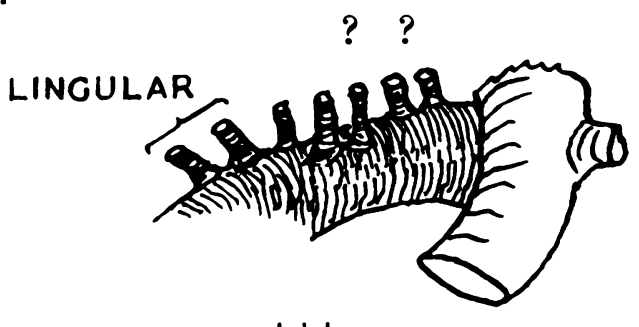

I lobe.

Type 19

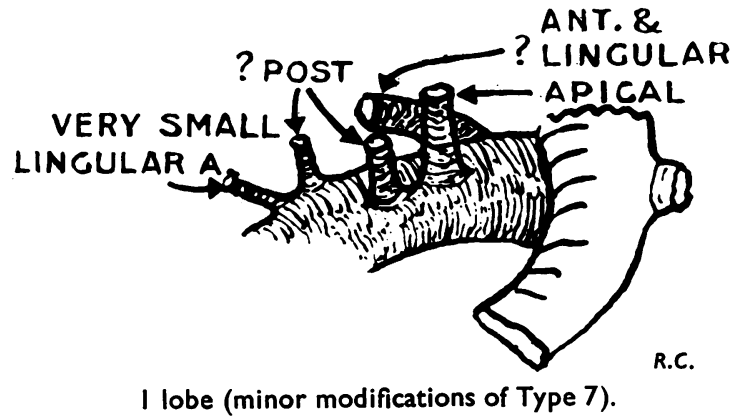

FIG. 7 (contd.)

With segmental stripping, from the hilum outwards, the anatomy may become obvious if the questionable vessels have not been divided first, but in cases where the vessels have to be dissected and divided before the segmental strip can be undertaken (and this happens commonly with fused structures) uncertainty may arise.

Attention should be drawn to some of the more unusual patterns. In Type 9 the posterior segmental artery arises below the lingular artery and runs upwards across the latter. This arrangement was found in two lobes. In Type 16 (one case) the lingula has a triple supply of which two vessels arise from a terminal division to the lower lobe. The artery to the posterior segment of the upper lobe also arises as a branch from the main lingular artery.

The same pattern of lingular supply is also found in Type 21 , but here there is a difference in the branches going to the superior segments.

Type 18 (one case) has such a large lingular artery as to lead to the belief that it must supply some of the anterior segment or perhaps the posterior segment. Type 19 (one case) would seem to be the reverse, with the anterior segmental artery giving a generous branch to the lingula. In 
Type 20

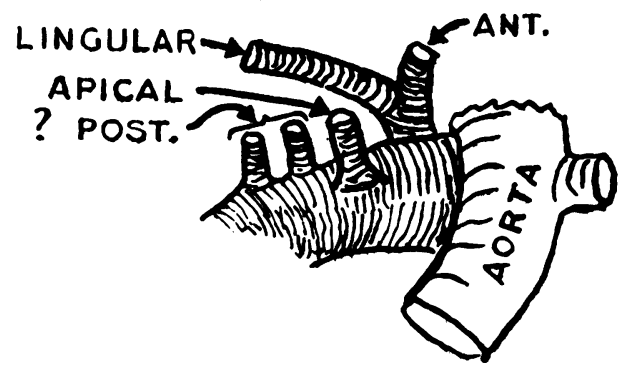

Type 22

I lobe.

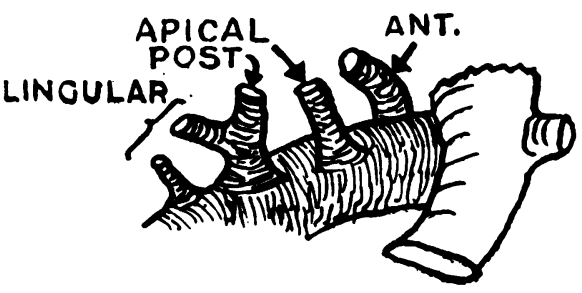

Type 24

I lobe.

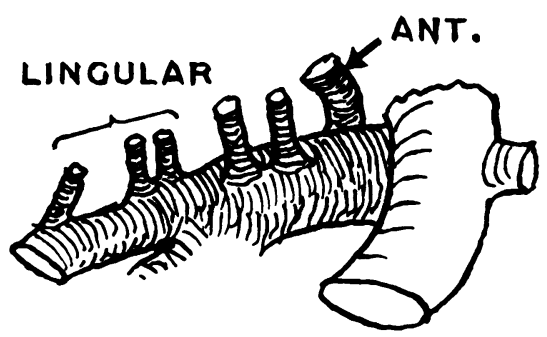

Type 26

I lobe.

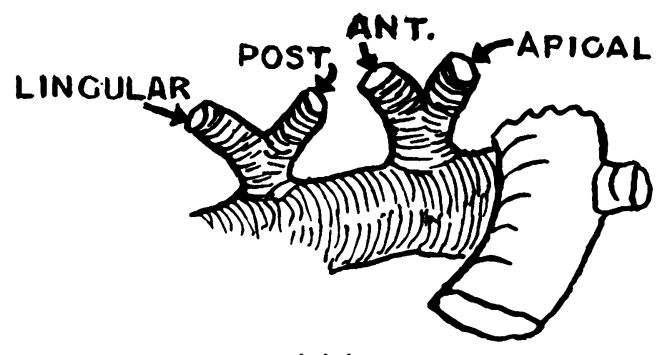

Type 28

I lobe.

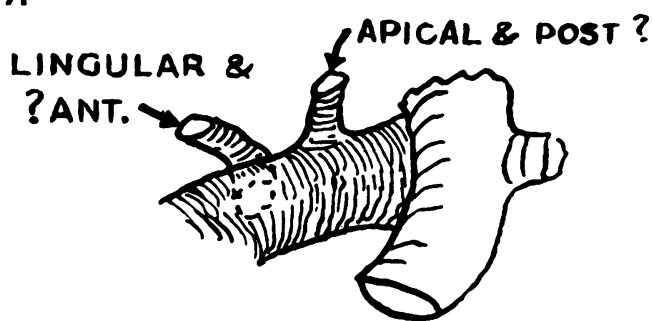

I lobe (lingular artery arising medially from pulmonary trunk).
Type 21

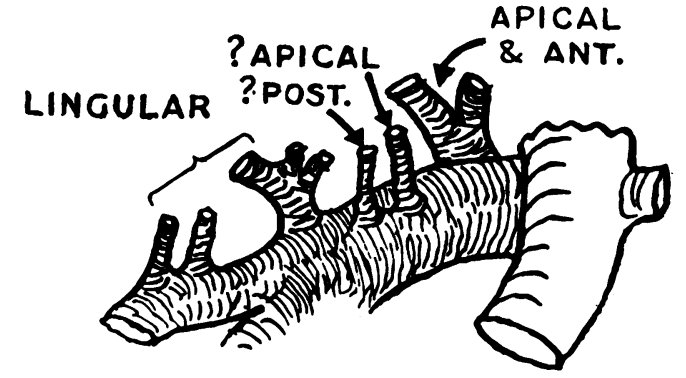

Type 23

I lobe.

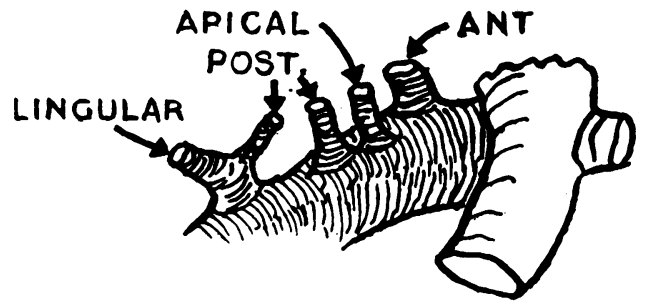

I lobe.

Type 25

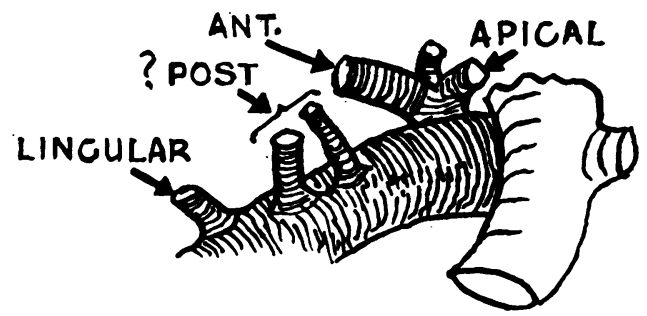

Type 27

I lobe.

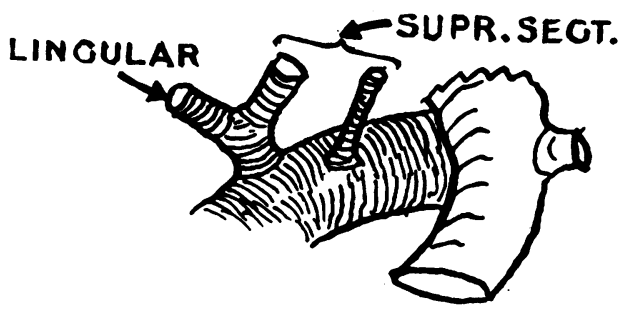

I lobe.

Type 29

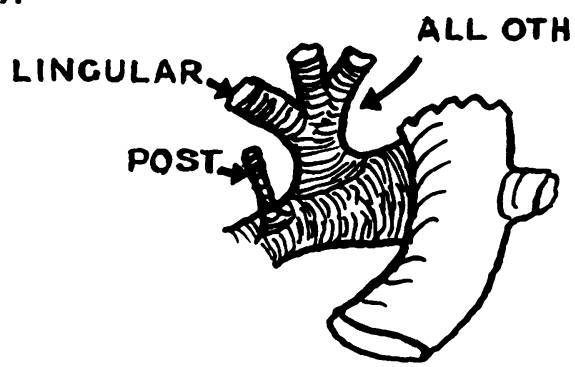

I lobe.

R.C.

FIG. 8.-Left upper lobe ( 107 lotes demonstrated). All seen from behind. 
Type 20 (one case), this supply of lingula from an anterior segmental vessel was confirmed, and in this type there was no other lingular artery.

Type 26 is simple but unusual, and if encountered in a lingulectomy might easily lead to trouble with the posterior segmental vessel if this originated further out and was not seen at an early stage in the dissection. In our single case it was readily recognizable for what it was.

Types 27, 28, and 29 are quite unusual. Fortunately they are also rare.

In seven of our 107 lobes the lingula received one or two vessels from the anterior terminal division to the lower lobe, and this arrangement should always be borne in mind. In our opinion the following summary holds good for about half of all cases :

The left upper lobe is most usually supplied by either three or four vessels. When there are three, the highest supplies the apical and anterior segments, the middle one the posterior segment, and the lowest the lingula. Type 2 illustrates this arrangement.

When four vessels are found they appear to be distributed as follows. The highest supplies the anterior segment and a part of the apical segment. The second supplies the rest of the apical segment. The third goes to the posterior segment, and the fourth to the lingula (Type 1).

Type 3 may reasonably be regarded as belonging to the general group with four vessels, but here it appears certain that the second branch supplies not only the apical segment but also a portion of the posterior segment. In other words, it is a true apical-posterior vessel. Such a welldefined apical-posterior artery only appeared in about $12 \%$ of our cases.

About $50 \%$ of left upper lobes fall into the combined Types 1, 2, and 3. While we agree generally with the classification, based on bronchial supply, which describes a compound apical-posterior segment on the left side, we must nevertheless point out that the arterial supply of the left upper lobe is often strongly suggestive of a separate posterior segment. From the surgical point of view, however, the apical and posterior segments are generally regarded as one, as in most segmental resections they have to be removed as one unit on account of the distribution of disease within them.

Left Lower Lobe (Secondary Hilum) (Fig. 9). -Fifty-seven left lower lobes were demonstrated, and among them we found four arterial patterns.

Type 1 was found in 44 of the 57 lobes $(77 \%)$. In it there is a single artery to the apical segment
Type I

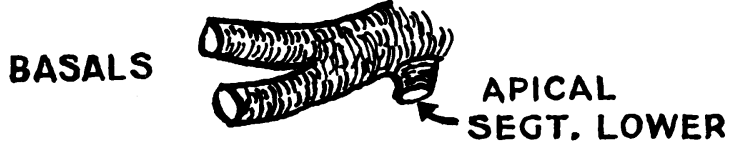

44 of 57 lobes demonstrated $=77 \%$.

Type 2

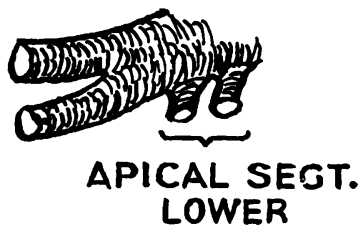

9 of 57 lobes demonstrated $=15 \%$.

Type 3

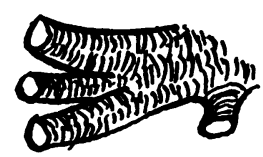

3 lobes.

Type 4

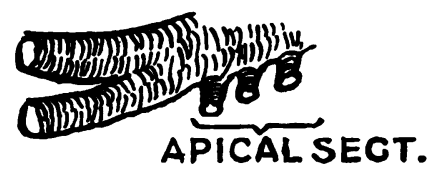

I lobe.

FIG. 9.-Left lower lobe (57 lobes demonstrated). Types 1 and 2 together account for $92 \%$ of all left lower lobe pulmonary arterial patterns found. All seen from behind.

of the lower lobe arising posteriorly, and just above the point at which the descending pulmonary arterial trunk divides into two terminal divisions. In Type 2 , nine lobes $(15 \%)$, there are two branches to the apical segment. These arise side by side and close together. Type 3 shows one apical segmental artery and three terminal divisions. This arrangement was found in three lobes. Type 4 (one lobe only) had three separate arteries to the apical segment. They all arose from the main stem before it split into two terminal divisions.

In no case was there an ascending apical segmental artery arising from a terminal division, as was found in two out of 43 lobes on the right side (Fig. 5, No. 4). 


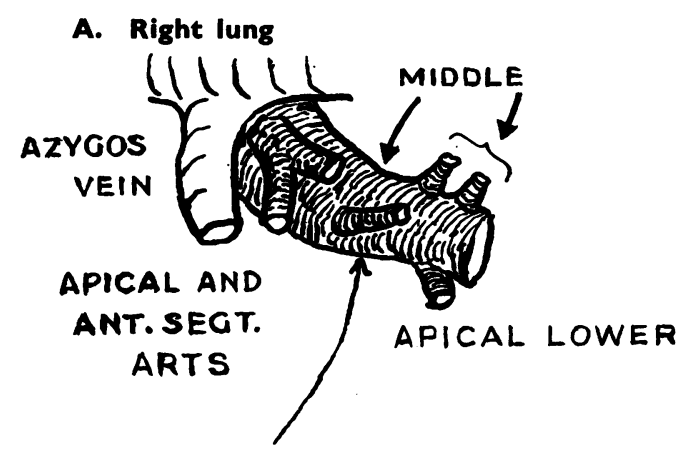

Usual posterior segment upper supplying abnormal middle lobe.

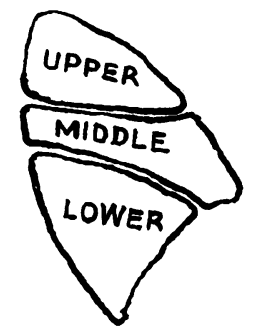

Lateral view of right lung:0 Posterior segment of upper? lobe is a part of middle $\overrightarrow{0}$ lobe with fully developedfissures. Middle lobe $\vec{\omega}$ extends back to posterions chest wall.

B. Left lung (three-lobed left lung in patient with dextrocardia).

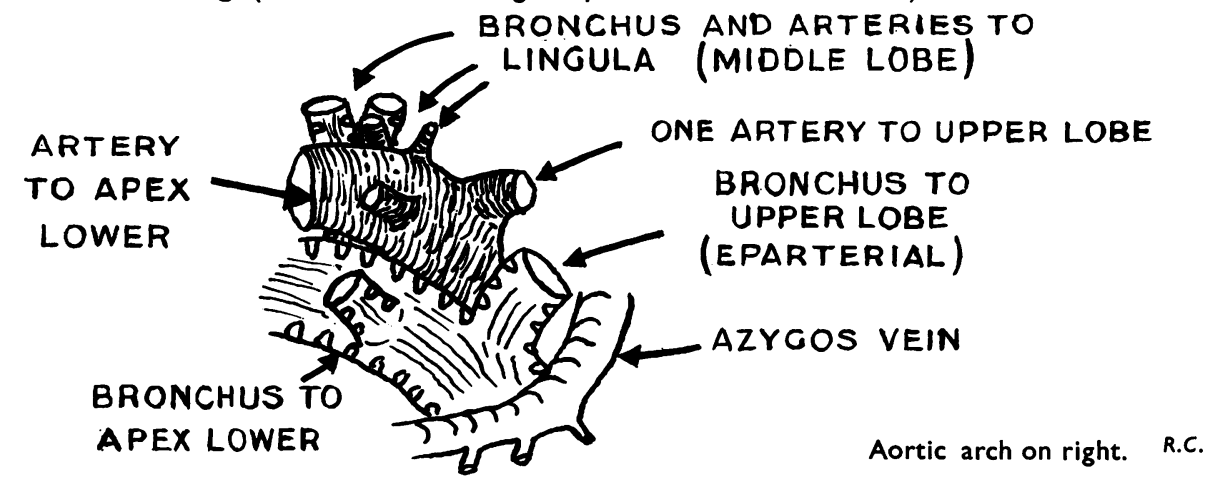

FIG. 10.-Two unusual patterns dictated by anatomical variations of the lung. Both above viewed from behind.

\section{Other Anomalies}

In any series of resections running into several hundreds some anatomical anomalies relating to the pulmonary tissue or the systemic vascular pattern can usually be found. There were six such in this series, and $t s$ they are of some surgical interest they will be briefly mentioned.

Fig. 10 shows the two variations of lung anatomy. In " A" is illustrated a right lung wherein the posterior segment of the upper lobe was an anatomical part of the middle lobe, being separated completely by a well-defined fissure from the upper lobe, which consisted of apical and anterior segments only. The middle lobe so formed was continuous from the posterior to the anterior chest wall, a most unusual picture and the only case in which we have come across it. The anterior part of this middle lobe possessed two arteries arising in the usual position from the anterior wall of the pulmonary artery. The posterior part derived its pulmonary arterial supply from a normally placed posterior segmental artery which ran downwards into this portion of the middle lobe (Fig. 10A). That it was in fact a representative of the posterior segmento of the upper lobe was confirmed by the fact that dissection of the upper lobe, after its removal,o revealed no trace of a posterior segmenta $\left.\right|_{x} ^{0}$ bronchus.

Fig. 10B was drawn from the appearance found. at operation on the left lung in a case of dextrocardia with a right-sided aortic arch. As $₹$ will be seen, there is a typical azygos vein on the? left side. The pulmonary artery emerges $>$ beneath it and lies anteromedially to a true eparterial bronchus supplying the upper lobe segments, as on the right side. The middle lobe was in the form of a lingula, but it had a true ${ }^{N}$ middle lobe bronchus arising separately from that going to the upper lobe.

The vascular pattern showed one large arterye for the upper lobe and two smaller ones for the middle lobe (lingula). This pattern is similar to? that shown for the right upper lobe. Type 20 (Fig. 2).

In Fig. 11 (1-4) have been recorded four systemic arterial anomalies, all of which were $\stackrel{\mathbb{Q}}{\Omega}$ found on the left side. In (1) there is a vesselo 
Type I

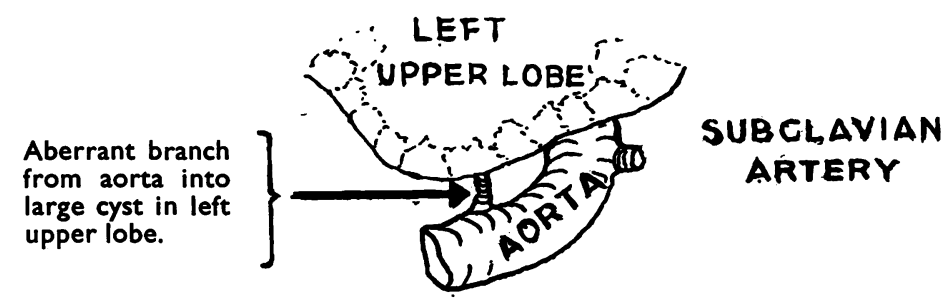

Type 2

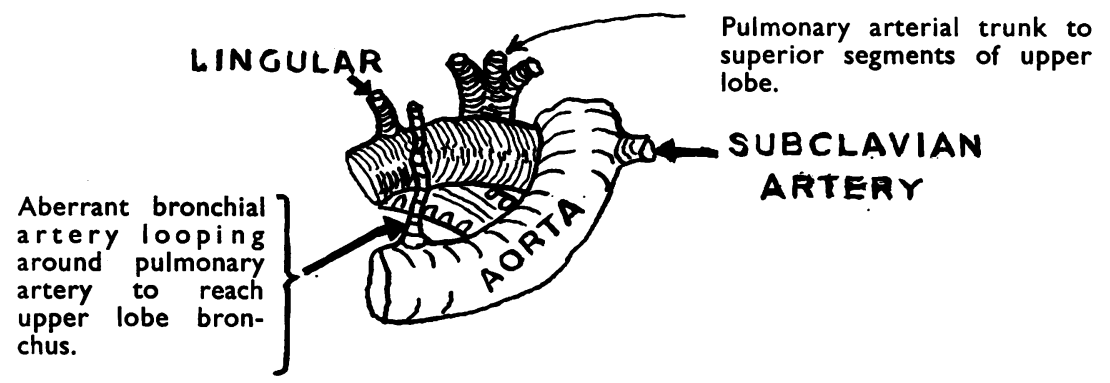

Type 3

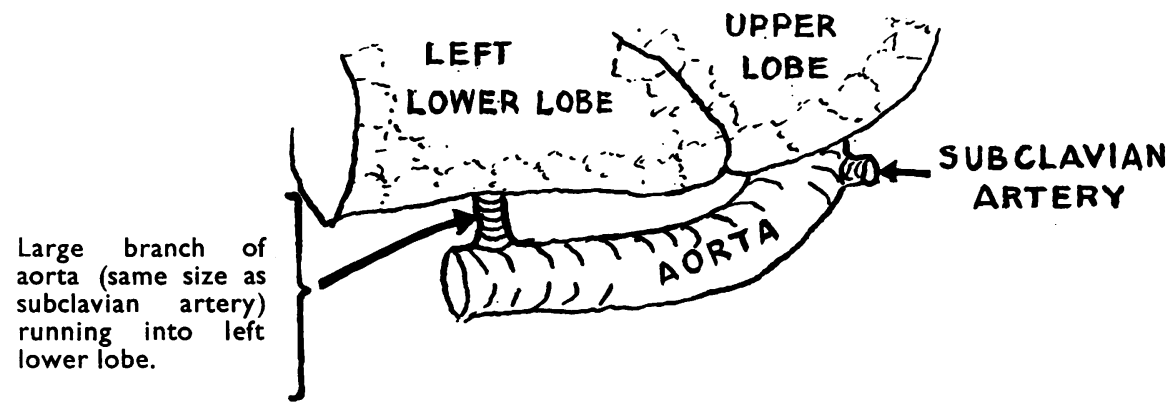

Type 4

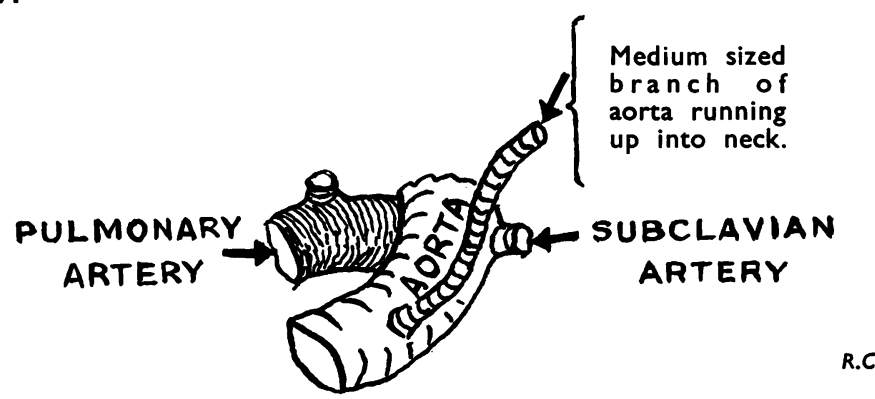

FIG. 11.-Systemic arterial anomalies found in 426 patients. The above four anomalies (one case each) were all found on the left side. All seen from behind. 
about 4 or $5 \mathrm{~mm}$. in diameter arising from the descending aorta, just below the arch, and running directly into a large cyst lying in the upper lobe. In (2) there is a systemic vessel arising from the same place as that in (1). This vessel, however, runs across the posterior, lateral, and anterior surfaces of the pulmonary artery to become a bronchial artery to the left upper lobe bronchus.

Normally all bronchial arteries run with the bronchus deep to the pulmonary artery, and not on its fissural surface. In (3) is shown a very large branch of the aorta arising about 2 in. above the diaphragm, and running directly into the lower lobe of the left lung. In this case the whole lobe was the site of extensive carnification and lipid degeneration, but whether it was a true sequestrated lobe was not determined. In (4) is shown a fairly large aortic branch arising from the arch distal to the subclavian artery and running forwards and upwards into the neck. Its final distribution was never ascertained, but its might have been going to the thyroid gland.

\section{SUMMARY}

Pulmonary arterial patterns and variations़ी have been displayed in 524 lungs or lobes af? operations for pulmonary resection on $426 \vec{\circ}$ patients. The pulmonary artery in the primary $\vec{L}$ lung hilum (34 right and 80 left) showed now variation.

The right upper lobe (152 cases) showed 14 variations, the right middle lobe (51 cases) fives variations, and the right lower lobe (43 cases) six variations. The left upper lobe (107 cases, showed no fewer than 29 differing arteriab patterns, and the left lower lobe $(57$ cases) only? four. In addition, two anomalies of lung tissue and four systemic arterial abnormalities were found and have been commented upon. 